\title{
Design of the Japanese Comprehensive Health-Economic Assessment for Appropriate Cardiac Imaging Strategy Including Outcome and Cost-Effectiveness in Stable Coronary Artery Disease Study (J-CONCIOUS)
}

\author{
Tomoaki Nakata, MD, PhD; Tomoyuki Takura, PhD; Hiroyoshi Yokoi, MD; \\ Kenichi Nakajima, MD, PhD; Shun Kohsaka, MD, PhD; \\ Akiyoshi Hashimoto, MD, PhD; Masato Nakamura, MD, PhD
}

\begin{abstract}
Background: The evaluation of stable coronary artery disease (SCAD) has evolved, and contemporary clinical practice guidelines emphasize the importance of in-depth consideration of procedure indications, risk stratification, and results of non-invasive imaging tests. However, little is known about the appropriate selection of imaging modalities for ischemia evaluation and the comparative cost-effectiveness in real-world clinical practice.

Methods and Results: The Japanese Comprehensive Health-Economic Assessment for Appropriate Cardiac Imaging Strategy including Outcome and cost-effectiveness in Stable Coronary Artery Disease Study (J-CONCIOUS), a multicenter observational study, was designed to prospectively enroll 2,500 patients with suspected or known SCAD, register clinical information and administrative records, and follow patients for 3 years. Any diagnostic or cardiac imaging modality (including stress tests using electrocardiography, echocardiography, or myocardial perfusion imaging; coronary computed tomographic angiography; and/or invasive coronary angiography with or without fractional flow reserve assessment) is acceptable. Clinical endpoints, such as all-cause mortality, cardiac death, and non-fatal myocardial infarction, will be obtained, along with quality of life assessment using the Seattle Angina Questionnaire. The cost-effectiveness of individual assessment patterns will be quantified by analysis of Diagnosis Procedure Combination (DPC) data, and quality-adjusted life years and the incremental cost-effectiveness ratio will be calculated.
\end{abstract}

Conclusions: J-CONCIOUS is expected to establish a risk-based and cost-effective imaging strategy for the detection and evaluation of functional myocardial ischemia and/or anatomical coronary imaging in Japan.

Key Words: Cardiac outcomes; Chronic coronary artery disease; Cost-effectiveness; Diagnostic modality; Functional ischemia

$\mathbf{T}$ he medico-economic burden - worldwide has been increasing because of an aging society, with an increase in the number of patients with atherosclerotic diseases. This has become particularly important given the increasing medical costs resulting from advances in diagnostic and therapeutic innovations. According to the Japanese Registry of All cardiac and vascular Diseases (JROAD), ${ }^{1}$ percutaneous coronary intervention (PCI) was performed in 296,743 patients with coronary artery disease
(CAD) in 2018 (234.7 cases/year per 100,000 members), consisting of elective PCI for stable CAD in $72.4 \%$ of patients and of emergency PCI for acute coronary syndrome (ACS) in the remaining patients. The number of PCI procedures has been increasing by $6.9 \%$ per year, in addition to the medical costs for these procedures, accounting for nearly $20 \%$ of national medical costs over the past 5 years (2012-2017) in Japan. ${ }^{2,3}$ However, in North America and Europe, PCI is preferentially performed in patients with

Received October 16, 2020; accepted October 16, 2020; J-STAGE Advance Publication released online November 17, 2020 Time for primary review: 1 day

Cardiology, Hakodate Goryoukaku Hospital, Hakodate (T.N.); Department of Healthcare Economics and Health Policy, Graduate School of Medicine, The University of Tokyo, Tokyo (T.T.); Cardiovascular Center, Fukuoka San-oh Hospital, Fukuoka (H.Y.); Department of Functional Imaging and Artificial Intelligence, Kanazawa University, Kanazawa (K.N.); Department of Cardiology, Keio University School of Medicine, Tokyo (S.K.); Department of Cardiology, Nephrology and Metabolism and Endocrinology, Sapporo Medical University School of Medicine, Sapporo (A.H.); and Division of Cardiovascular Medicine, Toho University Ohashi Medical Center, Tokyo (M.N.), Japan

T.T., S.K. are members of Circulation Reports' Editorial Team.

Mailing address: Tomoaki Nakata, MD, PhD, Cardiology, Hakodate Goryoukaku Hospital, 38-3 Goryoukaku-cho, Hakodate 040-8611, Japan. E-mail: tnakata@sapmed.ac.jp

All rights are reserved to the Japanese Circulation Society. For permissions, please e-mail: cr@j-circ.or.jp

ISSN-2434-0790 
ACS patients rather than in patients with stable CAD, with approximately two-thirds of PCI procedures for ACS and one-third for stable CAD.4,5 This is strongly based on definitive evidence for the prognostic benefits of this therapeutic intervention in ACS patients compared with stable CAD.

Over the past 13 years, multicenter clinical studies of stable CAD have clearly demonstrated that a non-invasive and/or functional ischemia assessment strategy, rather than an invasive or morphological stenosis-based strategy, can lead to greater improvements in patient outcomes by the initial introduction of optimal drug treatment appropriately combined with risk-based PCI selection. ${ }^{6-13}$ The fundamental goals of therapies for patients with stable CAD are to increase lifespan or to relieve angina. Present treatment options include revascularization by either PCI or coronary artery bypass grafting $(\mathrm{CABG})$ plus guidelinedirected medical therapy or initial management with guideline-directed medical therapy alone. The evaluation of stable CAD has also evolved, and contemporary clinical practice guidelines emphasize the importance of in-depth consideration of procedure indications, risk stratification, and results of non-invasive imaging tests. However, few contemporary large-scale data studies of the assessment of non-invasive imaging tests are available. It is critically important need to establish a clinically appropriate and prognostically cost-effective strategy in CAD patients.

In this context, a multicenter prospective cohort study was designed in Japan to establish a clinically appropriate and socioeconomically reasonable strategy, including diagnostic, therapeutic and prognostic management, in patients with known or suspected stable CAD in association with current drug treatment and coronary revascularization. Details of the study design are provided below.

\section{Methods}

\section{Study Title}

The title of this multicenter prospective cohort study is the Japanese Comprehensive Health-Economic Assessment for Appropriate Cardiac Imaging Strategy including Outcome and cost-effectiveness in Stable Coronary Artery Disease Study (J-CONCIOUS).

\section{Objectives}

The aim of this multicenter, observational, prospective cohort study is to establish a clinically appropriate and socioeconomically reasonable management strategy in patients with stable CAD by garnering diagnostic, therapeutic, and prognostic information, together with medical costs, in real-world clinical practice. The primary objective is to establish which diagnostic approach can most reasonably diagnose and risk stratify patients with stable CAD in terms of prognostic contribution leading to better patient outcomes and quality of life (QoL). The secondary objective is to reveal which diagnostic approach is most cost-effective in association with clinical outcomes and QoL.

\section{Clinical Endpoints}

Primary endpoints were defined as major adverse coronary events (MACEs), consisting of lethal (cardiac death, sudden cardiac death, fatal myocardial infarction) and non-lethal (e.g., non-fatal myocardial infarction and coronary revascularization by PCI or CABG) cardiac events. Secondary endpoints were defined as all-cause death, sudden death of unknown causes, hospitalization due to heart failure, and uncontrollable angina.

\section{Follow-up Protocol}

Patients enrolled in J-CONCIOUS will be followed-up prospectively for 3 years after obtaining written informed consent unless any lethal event is documented. Primary and secondary endpoints and QoL status will be confirmed regularly at least every year. QoL will be evaluated using the 5-level EQ-5D (EQ-5D-5L) ${ }^{\mathbf{1 4}}$ and the Seattle Angina Questionnaire (SAQ) Japanese version 7.0 at entry and during follow-up at regular intervals. The EQ-5D-5L is a QoL index that measures the utility value based on preference and is generally used for the evaluation of medical technology. The SAQ is a self-administered, diseasespecific health status questionnaire, ${ }^{15}$ and the SAQ angina frequency domain has been used in several studies as a secondary endpoint to quantify the long-term impact of alternative treatment strategies for ischemic heart disease. ${ }^{\mathbf{1 6}}$ After 12-months follow-up, patients will be divided into 2 groups according to therapeutic strategy (with or without PCI or CABG) for subsequent analysis.

\section{Inclusion Criteria}

To be eligible for inclusion in the study, patients need to meet any of the following criteria:

- Typical angina pectoris with all 3 of the following: chest discomfort or oppressive sensation, effort inducible, and easily relieved by rest and/or nitroglycerin use

- Atypical angina pectoris with 1 or 2 of the features described above for typical angina pectoris and at least 1 coronary risk factor strongly suggestive of stable CAD without the possibility of a non-cardiac disorder

- Instead of angina pectoris, effort-induced dyspnea or palpitation with coronary risk factors strongly suggestive of stable CAD without the possibility of a non-cardiac disorder

- Asymptomatic but electrocardiographic findings strongly suggestive of stable CAD.

In addition, patients must provide written informed consent.

\section{Exclusion Criteria}

Patients meeting any of the following criteria will be excluded from the study:

- Age $<20$ years

- ACS or suspected ACS

- A recent (within the past 12 months) history of CABG or PCI

- A recent (within the past 2 months) history of computed tomography coronary angiography (CTA) or invasive coronary angiography (CAG)

- A life expectancy <2 years because of malignancy

- Severe valvular heart disease requiring surgical repair or a history of surgery as a treatment for the condition

- Cardiomyopathy of non-ischemic or idiopathic etiology

- Advanced dementia, pulmonary disease, and/or allergic disease

- Systemic degenerative disorders or diseases that would interfere with a 3-year follow-up period

- Pregnancy or breastfeeding

- Deemed to be an inappropriate patient based on a participating physician's judgment. 


\begin{tabular}{|c|}
\hline $\begin{array}{l}\text { Inclusion Criteria for patients with known or suspected CAD } \\
\text { 1. Typical angina chest pain (all three features of typical angina) } \\
\text { 2. Atypical anginal chest pain (one or two features of typical angina) with coronary risks } \\
\text { 3. In stead of angina, effort-induced dyspnea and/or palpitation with coronary risks strongly } \\
\text { suggestive of stable CAD without possibility of non-cardiac disorder } \\
\text { 4. Asymptomatic but strongly suggestive of stable CAD on electrocardiographic findings }\end{array}$ \\
\hline $\begin{array}{l}\text { Registration of Clinical Data } \\
\text { History, coronary risks, laboratory, electrocardiographic and functional data }\end{array}$ \\
\hline $\begin{array}{l}\text { Registration of Non-Invasive or Invasive Imaging Tests } \\
\text { Exercise-stress electrocardiography, stress myocardial perfusion imaging with a radioactive tracer, } \\
\text { stress two-dimensional echocardiography, computed tomographic coronary angiography and/or selective } \\
\text { coronary angiography with or without fractional flow reserve assessment }\end{array}$ \\
\hline $\begin{array}{l}\text { Registration of Therapeutic Strategy } \\
\text { Just clinical course observation without any specific treatment, optimal medical treatment (OMT) and/or } \\
\text { elective coronary revascularization (PCI or CABG) }\end{array}$ \\
\hline $\begin{array}{l}\text { Prospective 3-year follow-up } \\
\text { Primary endpoints consisting of lethal and non-lethal cardiac events and QOL assessment }\end{array}$ \\
\hline
\end{tabular}

Figure. Study flowchart. CABG, coronary artery bypass grafting; CAD, coronary artery disease; OMT, optimal medical treatment; $\mathrm{PCl}$, percutaneous coronary intervention; QOL, quality of life.

\section{Sample Size}

This study plans to enroll nearly 2,500 patients with known or suspected stable CAD who meet the inclusion criteria. This Figure is based on an expected annual rate of $4 \%$ and an approximate number of $\geq 80$ events described above, and would enable us to perform a prognosis analysis in combination with a cost-effective analysis (CEA).

\section{Period for Patient Registration}

Patient registration will start within 6 months of study approval from an ethics committee and patients will then be followed-up prospectively. However, the study schedule can be changed if necessary.

\section{Diagnostic Modalities}

Current procedures available routinely in the cardiology practices at each medical facility are acceptable for this study, including exercise-stress electrocardiography (ECG), stress myocardial perfusion imaging (MPI) with a radioactive tracer, stress 2-dimensional echocardiography (2DE), CTA with or without fractional flow reserve (FFR) assessment, and invasive CAG with or without FFR assessment.

\section{Therapeutic Strategy}

Clinically standardized therapeutic approaches are accept- able for this study, including observation of the clinical course without any specific treatment, optimal medical treatment (OMT), and/or elective PCI and CABG.

\section{CEA}

The major target for data analysis is a CEA using data on MACEs, life years (LY), quality-adjusted LY (QALYs), and medical costs. This study plans to collect patients' overall medical costs using official insurance data for 3 years. CEA will be calculated as the ratio of the related medical costs over 3 years to LYs (US\$/LY) and QALYs (US\$/QALY). Despite the selection of deferral of PCI or $\mathrm{CABG}$ based on diagnostic imaging results, OMT has to be considered when patients have definitive stable CAD. Elective coronary revascularization scheduled during the follow-up period will also be included in the CEA. The index used for costs is the amount paid to medical facilities under the National Health Insurance system in Japan. It is planned that indirect costs will be excluded from this analysis because of a specific focus on direct medical costs from the public medical insurance database. Costs will be calculated by including initial and repeated examinations, guidance, testing and diagnosis, imaging and interpretation, prescriptions and medications, (drug) administration and injection, procedures and surgery, and recuperation and rehabilitation. 


\section{Statistical Analysis}

Subjects will be divided into 2 categories according to the initial diagnostic approaches. Category I will be made up of patients from Group 1 (a non-invasive strategy group) who have undergone stress ECG, stress MPI, stress 2DE, and/or CTA and patients from Group 2 (invasive strategy group), who have undergone CAG only. Category II will consist of patients from Group 3 (functional ischemia assessment group) who have undergone stress ECG, stress MPI, stress 2DE, and/or FFR combined with CTA and/or CAG and patients from Group 4 (morphology assessment group) who have undergone only CTA and/or CAG without functional ischemia assessment. Mean differences and patient backgrounds will be compared, and the independence of the relationship between costs and effects, will be evaluated using t-tests and Chi-squared tests as necessary. Kaplan-Meier analysis with a log-rank test will also be used. Statistical analyses will be performed using SPSS version 26.0 (IBM, New York, NY, USA). The level of statistical significance will be set at $5 \%$, and values will be expressed as the mean $\pm \mathrm{SD}$.

\section{Organization of the Study}

The 13 members of the Executive Committee (see Appendix 1), including a principal investigator and 2 sub-principal investigators, are responsible for the overall planning and execution of this study, including overseeing the protocol, providing guidance and any kind of support, and judging the inclusion and exclusion criteria for each patient. The staff office will be located at the Department of Healthcare Economics and Health Policy, The University of Tokyo. The staff office will supervise data collection, deidentification of individual data, protection of individual information, data analysis, and the presentation of analyzed data by producing an original J-CONCOIUS database. The staff office will be completely independent of the medical services in each of the participating hospital and of the funders.

\section{Data Availability}

The Executive Committee and staff office will supervise the following issues regarding data access and availability:

- Access to the dedicated database or individual deidentified participant data will be controlled: access to the data without an appropriate reason or committee approval will be limited, but data will be accessible based on requests from respective authors to correct their own data

- Analyzed data and related results will be shared until the final reports are officially published

- Any documents needed to perform J-CONCIOUS will be available anytime throughout the study.

\section{Research Ethics}

In accordance with the Declaration of Helsinki, the study design presented herein was planned by the J-CONCIOUS Executive Committee. On behalf of J-CONCIOUS, this study design was approved by the Institutional Review Board of Tokyo University Hospital and the Ethics Committee (Review No. 2018126NI) on January 30, 2019. It is also compulsory that approval be obtained from the ethics review boards of all participating institutions. Participating hospitals whose local ethics committee had officially approved this study up to August 29, 2020 are listed in Appendix 2. In accordance with the Declaration of Helsinki, all patients have to provide written informed consent to be enrolled in this study.

\section{Acknowledgments / Sources of Funding}

$\mathrm{J}-\mathrm{CONCIOUS}$ is grateful to Nihon Medi-Physics Co. Ltd (Tokyo, Japan) and FUJIFILM Toyama Chemical Co. Ltd (Tokyo, Japan) for their sponsorship. The funders have no role in this study, including study design, the collection, analysis, or interpretation of the data, manuscript writing, and the publication of the results.

\section{Disclosures}

T.T., S.K. are members of Circulation Reports' Editorial Team. T.T. has received grants from Nihon Medi-Physics Co. Ltd. The other authors have no conflicts of interest to declare.

\section{IRB Information}

This study was approved by the Institutional Review Board of Tokyo University Hospital and the Ethics Committee (Review No. $2018126 \mathrm{NI}$ ) on January 30, 2019.

\section{References}

1. JROAD (The Japanese Registry of All cardiac and vascular Diseases) [in Japanese]. National Cerebral and Cardiovascular Center Hospital. 2019. http://www.j-circ.or.jp/jittai_chosa/jittai_ chosa2016web.pdf (accessed July 1, 2020).

2. Takura T. Current trends in medical economics in the circulatory field: Socioeconomics background and research issue. Circ Rep 2019; 1: 342-346.

3. J-PCI Registry 2017 aggregate results [in Japanese]. Japanese Association of Cardiovascular Intervention and Therapeutics. 2018. http://www.cvit.jp/files/registry/annual-report/j-pci/2018. pdf (accessed July 1, 2020).

4. Fokkema ML, James SK, Albertsson P, Akerblom A, Calais F, Eriksson P, et al. Population trends in percutaneous coronary intervention: 20-year results from the SCAAR (Swedish Coronary Angiography and Angioplasty Registry). J Am Coll Cardiol 2013; 61: $1222-1230$.

5. Go AS, Mozaffarian D, Roger VL, Benjamin EJ, Berry JD, Blaha MJ, et al. Heart disease and stroke statistics - 2014 update: A report from the AHA. Circulation 2014; 129: e28-e292.

6. Tonino PA, Fearon WF, De Bruyne B, Oldroyd KG, Leesar MA, Ver Lee PN, et al. Angiographic versus functional severity of coronary artery stenoses in the FAME study fractional flow reserve versus angiography in multivessel evaluation. JACC Cardiovasc Interv 2010; 55: 2816-2821.

7. De Bruyne B, Fearon WF, Pijls NH, Barbato E, Tonino P, Piroth Z, et al. Fractional flow reserve-guided PCI for stable coronary artery disease. $N$ Engl J Med 2014; 371: 1208-1217.

8. Zimmermann FM, Ferrara A, Johnson NP, van Nunen LX, Escaned J, Albertsson P, et al. Deferral vs. performance of percutaneous coronary intervention of functionally non-significant coronary stenosis: 15-year follow-up of the DEFER trial. Eur Heart J 2015; 36: 3182-3188.

9. Boden WE, O'Rourke RA, Teo KK, Hartigan PM, Maron DJ, Kostuk WJ, et al. Optimal medical therapy with or without PCI for stable coronary disease. $N$ Engl J Med 2007; 356: 1503-1516.

10. Spertus JA, Jones PG, Maron DJ, O'Brien SM, Reynolds HR, Rosenberg Y, et al. Health-status outcomes with invasive or conservative care in coronary disease. N Engl J Med 2020; 382: $1408-1419$.

11. Fihn SD, Blankenship JC, Alexander KP, Bittl JA, Byrne JG, Fletcher BJ, et al. 2014 ACC/AHA/AATS/PCNA/SCAI/STS focused update of the guideline for the diagnosis and management of patients with stable ischemic heart disease: A report of the ACC/AHA Task Force on Practice Guidelines, and the AATS/ PCNA/SCAI/STS. JACC Cardiovasc Interv 2014; 64: 1929-1949.

12. Correction to: 2016 ACC/AHA guideline focused update on duration of dual antiplatelet therapy in patients with coronary artery disease: A report of the ACC/AHA Task Force on Clinical Practice Guidelines. Circulation 2016; 134: e192-e194.

13. Joseph J, Velasco A, Hage FG, Reyes E. Guidelines in review: Comparison of ESC and ACC/AHA guidelines for the diagnosis and management of patients with stable coronary artery disease. J Nucl Cardiol 2018; 25: 509-515.

14. Rabin R, de Charro F. EQ-5D: A measure of health status from the EuroQol Group. Ann Med 2001; 33: 337-343.

15. Spertus JA, Winder JA, Dewhurst TA, Deyo RA, Prodzinski J, 
McDonell M, et al. Development and evaluation of the Seattle Angina Questionnaire: A new functional status measure for coronary artery disease. JACC Cardiovasc Interv 1995; 25: $333-341$.

16. Cohen DJ, Van Hout B, Serruys PW, Mohr FW, Macaya C, den Heijer P, et al. Quality of life after PCI with drug-eluting stents or coronary-artery bypass surgery. N Engl J Med 2011; 364: $1016-1026$.

\section{Appendix 1}

The members of the J-CONCIOUS Executive Committee are listed below:

Principal Investigator

Tomoaki Nakata, MD, PhD (Hakodate, Japan)

Sub-Principal Lead Investigators

Tomoyuki Takura, PhD (Tokyo, Japan); Hiroyoshi Yokoi, MD,

$\mathrm{PhD}$ (Fukuoka, Japan)

Executive Committee Members

Kenichi Nakajima, MD, PhD (Kanazawa, Japan); Naoya Matsumoto,

$\mathrm{MD}, \mathrm{PhD}$ (Tokyo, Japan); Toshihiko Nishioka, MD, PhD (Saitama,

Japan); Nobuhiro Tanaka, MD, PhD (Tokyo, Japan); Masato

Nakamura, MD, PhD (Tokyo, Japan); Kazuya Takehana, MD, PhD

(Osaka, Japan); Hitoshi Matsuo, MD, PhD (Gifu, Japan); Taishiro
Chikamori, MD, PhD (Tokyo, Japan); Akiyoshi Hashimoto, MD, $\mathrm{PhD}$ (Sapporo, Japan); Shun Kosaka, MD, PhD (Tokyo, Japan)

\section{Appendix 2}

The J-CONCIOUS participating hospitals registered as of August 29, 2020 are:

Ehime Prefectural Central Hospital, Matsuyama; Kansai Medical University Hospital, Makikata; Kita-harima Medical Center, Ono; Gifu Heart Center, Gifu; Kin-ikyo Chuo Hospital, Sapporo; Kumamoto University Hospital, Kumamoto; Kumamoto Chuo Hospital, Kumamoto; Saiseikai Kumamoto Hospital, Kumamoto; Sapporo Medical University Hospital, Sapporo; Hospital of The University of Occupational and Environmental Health, Kita-kyushu; Shin Koga Hospital, Fukuoka; Kitano Hospital, Tazuke Kofukai Medical Research Institute, Osaka; Tokyo Medical University Hachioji Medical Center, Hachioji; Nihon University Hospital, Tokyo; Hakodate Goryoukaku Hospital, Hakodate; Hyogo College of Medicine College Hospital, Nishinomiya; Fukuoka Sanno Hospital, Fukuoka; Fukuoka University Faculty of Medicine, Fukuoka; Hokkaido Ohno Memorial Hospital, Sapporo; Urasoe General Hospital, Urasoe; Hiroshima City Hiroshima Citizens Hospital, Hiroshima; Teine Keijinkai Hospital, Sapporo; Hyogo Prefectural Awaji Medical Center, Awaji; Hyogo Brain and Heart Center, Himeji 